\title{
Trading strategy for decentralized energy resources in sequential electricity markets: A Swiss case study
}

\section{Conference Paper}

Author(s):

Han, Xuejiao; Kardakos, Evaggelos G.; Hug, Gabriela

Publication date:

2017

Permanent link:

https://doi.org/10.3929/ethz-b-000223881

Rights / license:

In Copyright - Non-Commercial Use Permitted

Originally published in:

https://doi.org/10.1109/ISGT-Asia.2017.8378322 


\title{
Trading strategy for decentralized energy resources in sequential electricity markets: A Swiss case study
}

\author{
Xuejiao Han, Evaggelos G. Kardakos, Gabriela Hug \\ Department of Information Technology and Electrical Engineering, ETH Zurich \\ Email: \{xuhan, ekardako, hug\}@eeh.ee.ethz.ch
}

\begin{abstract}
This paper derives optimal day-ahead trading strategies for an aggregator of a decentralized energy resources' mix, who participates in a multi-market environment, including a day-ahead, an intraday and a balancing market. The optimization problem is solved using multi-stage stochastic programming, which is subject to different levels of uncertainties such as variable generation output, day-ahead and intraday market prices. Risk management is conducted to investigate the effect of risk exposure on the total revenue of the aggregator. A case study based on the data from the Swiss electricity market demonstrates the effectiveness of the proposed model.
\end{abstract}

Index Terms-Decentralized energy resources (DER), electricity markets, mathematical programs with equilibrium constraints, price maker, probabilistic constraints.

\section{INTRODUCTION}

\section{A. Motivation and Goal}

The spot electricity market is a public financial market where electricity is traded on a short-term basis. It is organized as a sequence of markets, generally including a day-ahead (DA) market, several intraday (ID) markets and a balancing market (BM). In parallel, ancillary services markets are required to guarantee the secure operation of the grid in case of serious system contingencies. The spot market is gaining more and more importance, since it ensures the short-term optimization of procurement and supports the efficient integration of non-dispatchable energy resources in power markets. In recent years, the penetration of distributed energy resources (DERs), which are mainly renewable energy resources, has increased dramatically. However, DERs' participation in the market is still challenging due to the restrictions imposed by market regulations, their intermittent and uncertain nature and the lack of communication and cooperation among different DERs and system operators. These factors thus limit DERs' contributions to system operations and can cause high imbalance costs and low overall energy efficiency.

In order to fully utilize the value of flexibility potential of different DERs and enable them to engage in sequential electricity markets, coordinated dispatch and trading strategies at an aggregation level are of great interest.

\section{B. Related Works and Contributions}

A lot of work has been done in the field of optimal trading in sequential short-term markets, but most of them focuses on a single producer's point of view and on designing optimal trading strategies either for large-scale wind power plants or for wind producers coordinated only with energy storage

This work was supported by the Swiss Federal Office of Energy within SCCER FURIES and the Nexus project. technologies [1]-[5], ignoring the aggregation of different DER resources. In addition, several works develop trading strategies considering an incomplete market structure, for instance, [6] focuses on the DA and ID markets, while [7] presents strategies for participation only in DA and BM. As a result, there is a lack of studies dealing with optimizing coordinated trading strategies for small-scale DERs, which face limited possibilities for participating in electricity markets due to the barrier of requiring a minimum bidding quantity.

In this paper, we assume that DERs can participate in an electricity market including different trading floors through an aggregator, establishing an interface between small-scale DERs and system operators, following the principle of virtual power plants (VPP). VPPs provide an alternative way to enable the bidding of different types of DERs in power markets, by aggregation and provision of an overall operating profile. While most of the prior research on bidding strategies of VPPs focused on one specific spot market [8] or neglected the details of the market [9], [10], this paper aims to provide optimal trading strategies for a VPP considering its participation in multiple trading floors (e.g. DA market, ID market and BM).

Consequently, the contributions of this paper are as follows:

1) A stochastic optimization model is derived to determine optimal trading strategies for the DER aggregator, which groups different types of DERs such as storages, loads, variable and dispatchable generation units.

2) A joint optimization of different spot electricity markets using the multi-stage decision making is proposed. The model incorporates a reduced level of variable generation output uncertainty for the ID trading compared to the DA trading, since the ID market is cleared closer to the real-time delivery than the DA market.

3) Risk control is used to characterize the risk associated with profit variability, which stems from the uncertainty of different market prices and variable generation output.

The rest of the paper is organized as follows: the problem description is presented in Section II, and Section III focuses on the multi-stage stochastic programming method. The mathematical formulations and the proposed optimization model are presented in Section IV and V, respectively. Section VI provides an analysis for a case study based on Swiss market data, and finally conclusions are drawn in Section VII.

\section{PROBLEM DESCRIPTION}

In this paper, questions concerning the design of optimal DA trading strategies for an aggregated DER portfolio are 


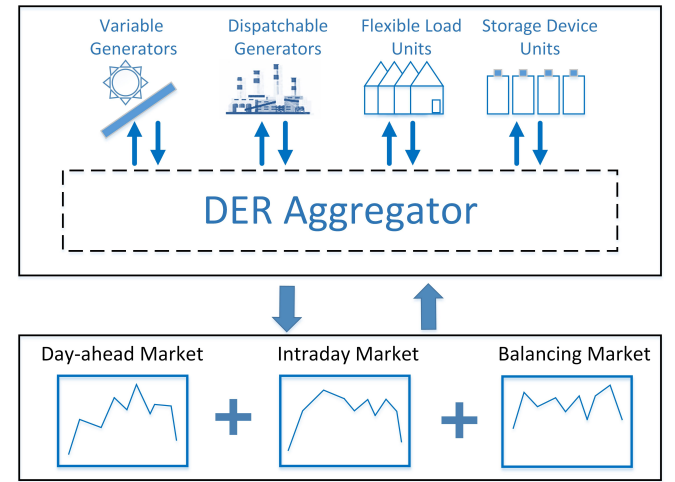

Fig. 1: Structure of the DER aggregator.

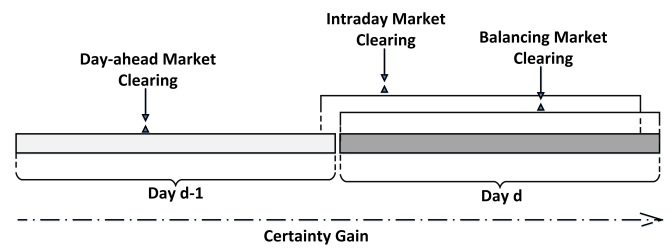

Fig. 2: Structure of sequential spot electricity markets.

addressed, enabling its participation in multiple electricity markets. Since DERs are often small-sized and even if participating as one virtual entity their decisions still have little impact on markets, the aggregator is assumed as a price-taker.

The aggregator is modeled as a cluster of storage devices, loads, dispatchable and variable generation units. It integrates the characteristics of diverse units into a single entity and generates a single dispatch portfolio. Aggregation is needed to take the advantage of different DERs and simultaneously enable their participation in power markets, in order to mitigate uncertainty problems and contribute to the provision of flexibility. The structure of the aggregator is shown in Fig. 1. The DER aggregator is assumed to participate in the Swiss electricity market that is organized around three major trading floors: the day-ahead, the intraday and the balancing market, as illustrated in Fig. 2, with the first two being organized by the European Power Exchange (EPEX) in Paris. According to regulations of EPEX, the Swiss DA market is cleared the day before at 11:00 offering the hourly product in a uniform auction for the following day, while the ID market is cleared continuously until one hour prior to the delivery time. In this paper, we consider a single ID auction that is cleared shortly before the delivery day (i.e. the day before at 23:00). To increase the flexibility of the market framework and to provide more opportunities for market participants to modify their schedules, additional ID auctions will be integrated in future extensions of this work. Finally, the balancing market is designed to compensate the difference between the scheduled and the measured energy and it is cleared after the realizations of supply and demand [11]. In this context, it is assumed that all imbalances can be corrected through the balancing market.

The objective of this work is to investigate the combined optimization of a DERs' mix in sequential electricity markets, considering different sources of uncertainty.

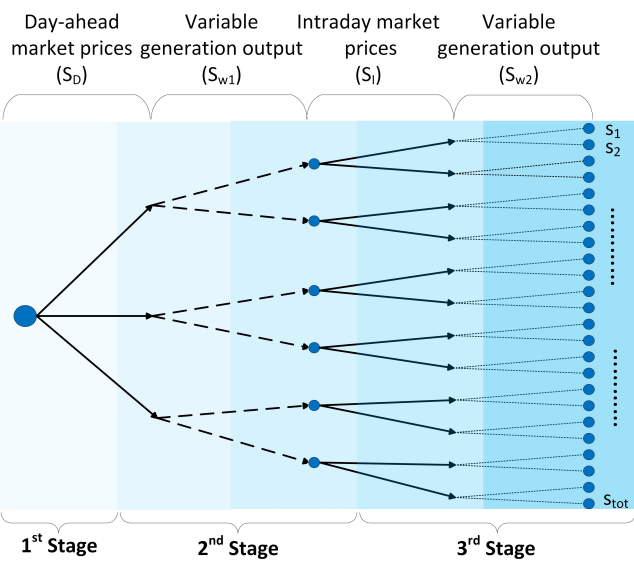

Fig. 3: Multi-stage stochastic optimization model.

III. Multi-Stage Stochastic Programming

As the aforementioned problem is subject to different levels of uncertainties such as market prices and variable generation outputs, the stochastic programming framework needs to be used. Since the most straightforward way to model a stochastic process is to use scenarios, in this model, a set of scenarios characterizing different sources of uncertainties is developed based on the following structure [12]:

1) Generate $S_{D}$ scenarios for day-ahead market prices.

2) For each DA price scenario, generate $S_{w 1}$ scenarios for variable generation output between closures of DA and ID markets, i.e. for the hours 11:00 to 23:00 of day d-1.

3) For each variable generation output scenario between DA and ID market, generate $S_{I}$ scenarios for ID prices.

4) For each ID price scenario, generate $S_{w 2}$ scenarios for variable generation output at real-time delivery, i.e. for the hours 0:00 to 24:00 of day d.

Thus, we have in total $S_{t o t}=S_{D} \times S_{w 1} \times S_{I} \times S_{w 2}$ scenarios, which are assumed to have the same probability of realization. As the information is revealed sequentially, this scenario tree is fed into a multi-stage stochastic optimization model shown in Fig. 3, where nodes and arcs represent decision stages and realizations of stochastic variables, respectively.

It is important to mention the non-anticipativity characteristic of the multi-stage stochastic programming formulation, which means that if realizations of stochastic processes are identical up to stage $k$, then their decision variables must be identical up to that stage [12]. The structure of the proposed multi-stage optimization model is as follows [2], [7], [13]:

- 1st stage: In electricity markets, producers and consumers must decide their offer and demand curve, respectively. As a prosumer, the aggregator submits its DA offer and demand curves that consist of the price-quantity pairs (one for each DA price scenario) derived by the solution of the proposed problem. Both curves are unique (scenario independent) for each hour of the scheduling period, i.e. they are the same for all possible realizations of stochastic variables in the second and the third stage.

- 2nd stage: After the revelation of the DA market price, the accepted quantity of the DER aggregator is known (based 


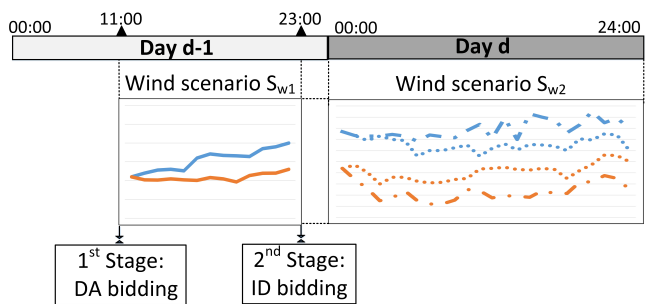

Fig. 4: Certainty gain effect regarding wind forecast.

on the submitted offer/demand curve). Then after the closure of the DA market and shortly before the closure of the ID market, the aggregator updates the forecast of its variable generation output for the hours 0:00 to 24:00 for the delivery day d considering the information newly arrived between the DA and ID market, i.e. the realized variable generation output between 11:00 and 23:00 of day d-1. Based on the updated forecast and the realized DA price-quantity bid of the previous stage, the aggregator submits new offer and demand curves to the ID market so as to correct its resources' schedules. ID bids are dependent on the second-stage scenarios, i.e. the DA market prices, but they are unique and independent of the scenario realizations at the third decision stage, i.e. the ID market prices and the variable generation output at real-time delivery.

Note that when the aggregator submits bids into the DA market at 11:00 for day (d-1), it has to forecast the wind output from 00:00 to 24:00 of the following day (day d), 13-37 hours in advance. When bidding into the ID market, the historical wind output between the closure of the DA market and the ID market (i.e. 11:00-23:00 for day d-1) is realized, and thus can be used to improve the hourly wind forecast for the delivery day. In other words, the aggregator bids into the ID market with a reduced wind uncertainty level than that for the DA market. This certainty gain effect [12] is visualized in Fig. 4 [7].

- 3rd stage: After the revelation of the ID market price, the accepted quantity of the aggregator for the ID market is known. Then the DER aggregator decides on the operation schedule of the dispatchable generating units, loads and storage devices once the variable generation output, DA and ID market prices are known.

\section{Constraints Modelling}

The DER aggregator consists of four different types of components, namely loads, storage devices, dispatchable and variable generation units as shown in Fig. 1. It participates in day-ahead, intra-day and balancing markets. To optimize the trading strategy of the aggregator, all types of devices as well as how they are coordinated and the market environment in which they participate need to be modeled.

\section{A. Dispatchable Generation Unit}

Dispatchable generation is modeled by a power output $P_{t, s}^{G}$ at time $t$ for scenario $s$, which is limited by maximum/minimum power output $P^{G, \max }$ and $P^{G, \min }$, and the maximum ramp rate $r^{\max }$, i.e.

$$
\begin{array}{r}
u_{t, s} P^{G, \text { min }} \leq P_{t, s}^{G} \leq u_{t, s} P^{G, \max } \\
\left|P_{t, s}^{G}-P_{t-1, s}^{G}\right| \leq r^{\max }
\end{array}
$$

where $u_{t, s}$ is a binary variable denoting the on/off status of the generator. The startup and shut down statuses are denoted by two binary variables $y_{t, s}$ and $z_{t, s}$, which are restricted by

$$
\begin{aligned}
& y_{t, s}-z_{t, s}=u_{t, s}-u_{t-1, s} \\
& y_{t, s}+z_{t, s} \leq 1
\end{aligned}
$$

ensuring that the dispatchable generation unit may not operate in startup and shut down mode simultaneously. The operating $\operatorname{cost} C_{t, s}^{O P}$ and the startup (shut down) $\operatorname{cost} C_{t, s}^{S}$ are approximated with linear functions

$$
\begin{aligned}
C_{t, s}^{O P} & =c_{o p} \cdot P_{t, s}^{G} \\
C_{t, s}^{S} & =c_{s} \cdot\left(y_{t, s}+z_{t, s}\right)
\end{aligned}
$$

where $c_{o p}$ and $c_{s}$ are constant.

\section{B. Storage Device}

A generic battery model is used. The minimum and maximum state of charge (SOC) of the battery are defined by $E^{S, \min }$ and $E^{S, \max }$, respectively, while the maximum inflow and outflow of the storage are $P^{S C, \max }$ and $P^{S D, \max }$, respectively. This results in the following set of equations:

$$
\begin{aligned}
E^{S, \text { min }} & \leq E_{t, s}^{S} \leq E^{S, \max } \\
0 & \leq P_{t, s}^{S C} \leq P^{S C, \max } \\
0 & \leq P_{t, s}^{S D} \leq P^{S D, \text { max }}
\end{aligned}
$$

where $E_{t, s}^{S}, P_{t, s}^{S C}$ and $P_{t, s}^{S D}$ indicate the SOC, charging and discharging power of the battery at time $t$ under scenario $s$, respectively. The end storage level of the battery $E_{T, S}^{S}$ is set to be equal to the initial level $E_{0}^{S}$ Finally, the relationship of storage levels for two consecutive time steps are defined by

$$
E_{t, s}^{S}=E_{t-1, s}^{S}+\eta P_{t, s}^{S C} \Delta t-\eta^{-1} P_{t, s}^{S D} \Delta t
$$

where $\eta$ indicates battery's conversion efficiency.

\section{Load}

It is assumed that parts of the aggregator's loads are flexible and participate in the demand response (DR) program through load shifting from the initial load forecast $P_{t}^{L, \text { est }}$. The final scheduled load profile is given by

$$
P_{t, s}^{L, s c h}=P_{t}^{L, e s t}+r_{t, s}^{L u}-r_{t, s}^{L d}
$$

where $r_{t, s}^{L u}$ and $r_{t, s}^{L d}$ indicate the load increase and decrease for time step $t$, respectively. The values are determined based on the maximum load allowed to be shifted denoted by $P^{L, D R}$ (defined by the DR contract signed between the aggregator and customers), the maximum energy consumption $P^{L s h, \max }$ (i.e. capacity) of the flexible load, and the current energy consumption $P_{t}^{L s h}$ of the flexible load. Mathematically, $r_{t, s}^{L u}$ and $r_{t, s}^{L d}$ are calculated based on the following equations [13]:

$$
\begin{aligned}
0 \leq & r_{t, s}^{L u} \leq \min \left(P^{L, D R} \cdot u_{t, s}^{L u},\left(P^{L s h, \text { max }}-P_{t}^{L s h}\right) \cdot u_{t, s}^{L u}\right) \\
0 \leq & r_{t, s}^{L d} \leq \min \left(P^{L, D R} \cdot u_{t, s}^{L d}, P_{t}^{L s h} \cdot u_{t, s}^{L d}\right) \\
& u_{t, s}^{L d}+u_{t, s}^{L u} \leq 1
\end{aligned}
$$

where $u_{t, s}^{L u}$ and $u_{t, s}^{L d}$ are binary variables indicating the load increase or decrease. The last equation ensures that only one 
of $u_{t, s}^{L u}$ or $u_{t, s}^{L d}$ is 1 . Furthermore, the total daily shifted load defined as the sum of absolute values of the daily load decrease and increase is restricted by an upper limit, i.e.

$$
\sum_{t}\left(r_{t, s}^{L u}+r_{t, s}^{L d}\right) \Delta t \leq E_{\max }^{L, s h}
$$

Additionally, it is required that the energy consumption during the scheduling horizon ( 24 hours) should not be changed, i.e.

$$
\sum_{t}\left(r_{t, s}^{L u}-r_{t, s}^{L d}\right)=0
$$

Under the assumption that the DR program is based on long-term contracts with customers, the cost of changing the demand profile is neglected.

\section{Electricity Markets}

1) Day-ahead Market: We decompose the DA bidding curve $\left(P_{t, s}^{D A}, p r_{t, s}^{D A}\right)$ into an offer curve $\left(P_{t, s}^{D A+}, p r_{t, s}^{D A}\right)$ and a demand curve $\left(P_{t, s}^{D A-}, p r_{t, s}^{D A}\right)$, i.e.

$$
P_{t, s}^{D A}=P_{t, s}^{D A+}-P_{t, s}^{D A-}
$$

where $p r^{D A}$ is the price, and $P^{D A+}, P^{D A-}$ are the supply and the demand in the DA market. The non-decreasing (nonincreasing) character of the resulting offer (demand) curve is ensured by

$$
\begin{array}{lll}
P_{t, s}^{D A+} \leq P_{t, s^{\prime}}^{D A+} & \forall t, \forall s, s^{\prime}: & p r_{t, s}^{D A} \leq p r_{t, s^{\prime}}^{D A} \\
P_{t, s}^{D A-} \leq P_{t, s^{\prime}}^{D A-} & \forall t, \forall s, s^{\prime}: & p r_{t, s}^{D A} \geq p r_{t, s^{\prime}}^{D A}
\end{array}
$$

The non-anticipativity of the DA decision is enforced by

$$
\begin{array}{lll}
P_{t, s}^{D A+}=P_{t, s^{\prime}}^{D A+} & \forall t, \forall s, s^{\prime}: & p r_{t, s}^{D A}=p r_{t, s^{\prime}}^{D A} \\
P_{t, s}^{D A-}=P_{t, s^{\prime}}^{D A-} & \forall t, \forall s, s^{\prime}: & p r_{t, s}^{D A}=p r_{t, s^{\prime}}^{D A}
\end{array}
$$

which forces the DA decisions at the second stage to be equal in all scenarios with identical realizations of random variables up to the second stage. In other words, the hourly DA offer (demand) curve is unique, irrespective of the market price and variable generation output realizations [12].

2) Intraday Market: The intraday market is modeled in a similar way as the day-ahead market, except that the ID market clearing is closer to real-time delivery and exposed to a reduced level of variable generation output uncertainty. The hourly ID offer (demand) curve submitted to the ID market at the third stage is again unique, i.e. independent of the realizations of ID market prices and variable generation outputs at real-time delivery. Consequently, the ID decisions (points on the offer (demand) curve) at the third stage should be equal in all scenarios with identical realizations of DA prices and variable generation outputs between the DA and the ID market. This is modeled as

$$
\begin{array}{rr}
P_{t, s}^{I D+}=P_{t, s^{\prime}}^{I D+} \quad \forall t, \forall s, s^{\prime}: & p r_{t, s}^{D A}=p r_{t, s^{\prime}}^{D A} \\
\forall t \in\left[t_{1}, t_{2}\right]: \quad P_{t, s}^{W}=P P_{t, s^{\prime}}^{W} \\
\forall t, \forall s, s^{\prime}: \quad p r_{t, s}^{I D}=p r_{t, s^{\prime}}^{I D} \\
P_{t, s}^{I D-}=P_{t, s^{\prime}}^{I D-} \quad \forall t, \forall s, s^{\prime}: \quad p r_{t, s}^{D A}=p r_{t, s^{\prime}}^{D A}, \\
\forall t \in\left[t_{1}, t_{2}\right]: \quad P_{t, s}^{W}=P P_{t, s^{\prime}}^{W} \\
\forall t, \forall s, s^{\prime}: \quad p r_{t, s}^{I D}=p r_{t, s^{\prime}}^{I D}
\end{array}
$$

where $P_{t, s}^{W}$ is the variable generation output.
According to the data from [11], the total energy traded in the Swiss DA market in 2015 amounted to 22.91 TWh, while the volume of the ID market for the same year is equal to only 1.44 TWh. Based on this fact, a parameter $\gamma_{I D}$ is defined to limit the trading volume in the ID market according to

$$
\sum_{t} P_{t, s}^{I D} \leq \gamma_{I D} \sum_{t} P_{t, s}^{D A} \quad \forall s
$$

3) Balancing Market: The balance energy price mechanism in Switzerland is a two-price system in which prices for balance energy are classified according to the deviation direction of a balance group. Balancing market prices are modeled in a similar way with a base price and a percentage of DA market prices. The price of the positive imbalance $P_{t, s}^{B+}$, i.e. the price of selling electricity in the BM in the case of surplus generation or deficit consumption, is defined by $p r_{t, s}^{B+}$ and the price of the negative imbalance $P_{t, s}^{B-}$, i.e. the price of purchasing electricity in the BM under the situation of deficit generation or surplus consumption, is defined by $p r_{t, s}^{B-}$. Note that the BM price is dependent on the day-ahead price and therefore the uncertainty concerning the BM price is not considered. The following equation ensures that the aggregator can only sell (purchase) electricity in the BM at a price lower (higher) than the DA market price [11]:

$$
\begin{array}{ll}
p r_{t, s}^{B+}=a_{1} \cdot\left(p r_{t, s}^{D A}+P_{1}\right) & \forall t, \forall s \\
p r_{t, s}^{B-}=a_{2} \cdot\left(p r_{t, s}^{D A}-P_{2}\right) & \forall t, \forall s
\end{array}
$$

where $a_{1}, a_{2}, P_{1}, P_{2}$ are all positive constants with $a_{1} \geq 1$ and $0 \leq a_{2} \leq 1$.

\section{E. Aggregator's Portfolio}

The DER aggregator along with the sequential markets (i.e. DA, ID, BM) constitute a closed energy system that must be balanced at each time period $t$. This means that the amount of energy that is generated from both dispatchable and stochastic power plants and drawn from the storage units minus the amount of energy that is consumed by the load and used to charge the storage units must be equal to the exchange in spot electricity markets for each time period $t$, i.e.

$$
P_{t, s}^{G}+P_{t, s}^{S D}+P_{t, s}^{W}-P_{t}^{L, s c h}-P_{t, s}^{S C}=P_{t, s}^{D A}+P_{t, s}^{I D}+P_{t, s}^{B}-P_{t, s}^{B-}
$$

\section{Formulation of Optimization Problem}

The profit of the aggregator comprises the revenues from participating in the day-ahead market, the intraday market and the balancing market minus generation costs. Mathematically,

$$
\begin{array}{r}
R=\sum_{t} \sum_{s} \pi_{s}\left(p r_{t, s}^{D A} \cdot P_{t, s}^{D A}+p r_{t, s}^{I D} \cdot P_{t, s}^{I D}-\right. \\
\left.p r_{t, s}^{B-} \cdot P_{t, s}^{B-}+p r_{t, s}^{B+} \cdot P_{t, s}^{B+}-C_{t, s}^{O P}-C_{t, s}^{S}\right)
\end{array}
$$

where $\pi_{s}$ represents the probability of scenario $s$. Note that only generation costs of the conventional units are considered, while the generating costs of stochastic generators and storage devices are assumed to be zero.

Additionally, trade-offs have to be made between the expected profit and the impact of uncertainty. Hence, the objective function consists of two parts: one maximizing the 
expected profit of the aggregator, and the other minimizing the risk exposure to uncertainties by the $\mathrm{CVaR}$ (Conditional Value at Risk) [14] of the expected revenue, i.e.

$$
\max \left\{(1-\beta) R+\beta\left(\zeta-\frac{1}{1-\alpha} \sum_{s} \pi_{s} \zeta_{s}\right)\right\}
$$

where $\beta$ is a weighting parameter with $\beta \in[0,1] ; \alpha$ is the confidence level with $\alpha \in(0,1) ; \zeta$ and $\zeta_{s}$ are auxiliary variables restricted by

$$
\begin{aligned}
\zeta-R_{s} & \leq \zeta_{s} \\
\zeta_{s} & \geq 0
\end{aligned}
$$

in which $R_{s}$ is the expected revenue of scenario $s$ with $\sum_{s} R_{s}=R$.

The constraints of the optimization problem correspond to the constraints given by different components of the aggregator (i.e. storage, load, dispatchable and variable generation), different electricity markets, as given in (1)-(16) in Sec. III, and the constraints concerning risk control given in (19).

\section{CASe Study}

A. Data

All DER units considered are aggregated and consist of several individual generators, loads or storage devices. The aggregated dispatchable generation unit has a maximum and minimum output limit of $100 \mathrm{MW}$ and $10 \mathrm{MW}$, whereas the ramp rate limit is $25 \mathrm{MW}$ per hour. The minimum/maximum capacity of the aggregated storage unit are set to $20 \mathrm{MWh}$ and $240 \mathrm{MWh}$, respectively. The charging and discharging limit is $120 \mathrm{MW}$, both storage levels at the beginning and at the end of the day are $120 \mathrm{MWh}$. The conversion efficiency of storage devices is 0.9. Parameters of loads and the DR program are taken from the example in [13].

Market price and wind output forecasting are outside the scope of this paper. The market price data are obtained from the European Power Exchange (EPEX), and are publicly available in [15]. These data correspond to the prices that occurred on the Tuesdays from August 2016 to September 2016. Parameters for the balancing market price are $a_{1}=1.2$, $a_{2}=0.8, P_{1}=1 \mathrm{ct} / \mathrm{kWh}$ and $P_{2}=0.5 \mathrm{ct} / \mathrm{kWh}$. The wind output scenario for the same season is based on the simulated wind speed data of Zurich ( $\mathrm{N} 47^{\circ} 22^{\prime}$ E $8^{\circ} 33^{\prime}$ ) generated by Renewables.ninja [16]. To convert wind speed into wind power, the power curve for a popular small wind turbine in Switzerland (model Aventa AV-7 (6.5 kW)) is used [17]. An aggregated wind power plant unit consisting of 30 Aventa AV7 wind turbine models is modeled. The number of DA price, ID price and real-time variable generation output scenarios are six, six and four respectively.

We first carried out the multi-stage optimization with the ID trading limit $\gamma_{I D}=10 \%$, considering a complete market structure, including DA, ID and BM. This is considered as a base case and the results for a single scenario are discussed as an example in the following subsection. Then we investigated effects of the multi-market environment and limited ID market capacity by carrying out multi-stage optimizations with different market structures and ID trading limits. In the end,

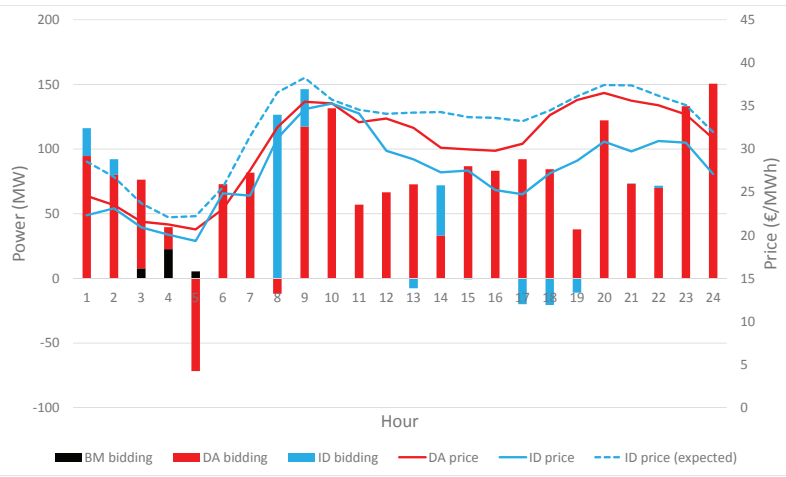

(a) Market trading analysis

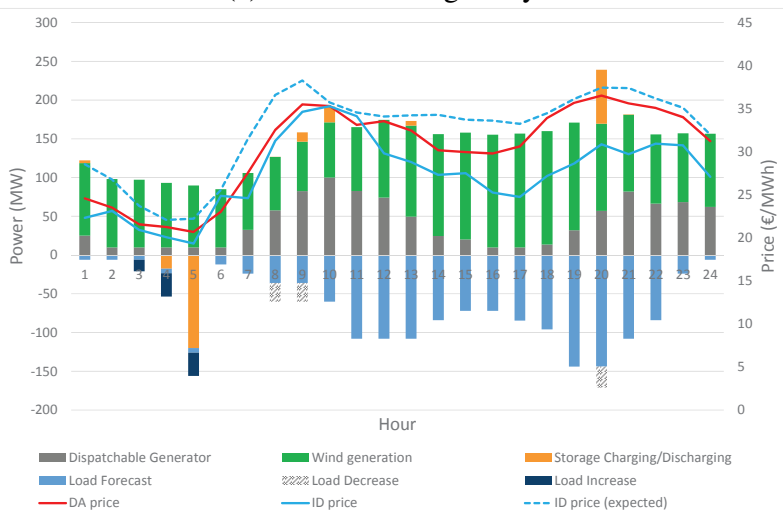

(b) DERs operations analysis

Fig. 5: Single scenario analysis for relatively high wind.

the effect of risk control is analyzed by integrating the CVaR into the objective function of the base case.

\section{B. Single Scenario Analysis}

Fig. 5 shows the bidding strategy and the electricity profile for the aggregator of an illustrative scenario with high wind. It is seen that generally the aggregator trades in markets according to the "buy low, sell high" strategy. Following the formulated multi-stage stochastic programming, the cleared DA bidding quantity for each scenario realized at the second stage is based on the realized DA price, the expected generation output and the expected ID price. That is to say, the aggregator bids into the DA market without knowing the ID price and makes decisions mainly based on the realized DA price and the expected ID price. For example in hour 8 , when the difference between the realized DA price (red curve) and the expected ID price (dotted blue curve) is the biggest, the aggregator sells all the energy in the ID market even though the DA price for that hour is relatively high compared to other periods of the day. On the other hand, when bidding into the ID market for each ID price scenario, the realized DA price, the realized ID price and the expected generation output will be considered. The ID market serves as an additional trading floor for the aggregator to balance its positions in response to newly arrived wind forecast, and simultaneously it provides an opportunity for the aggregator to make profits by taking advantage of DA and ID market price differences [12]. Since BM prices are 
always unfavorable to DA market prices, it is only used for covering deviations between scheduled electricity and actually produced electricity. Due to the integration of different trading floors and the coordination of different DERs, imbalances are limited in most of the scenarios. Figure (5b) shows that both the storage device and the dispatchable generation unit operate mainly in response to market prices and wind output level. It is also notable that even when market prices are lower than the generation cost $(28 € / \mathrm{MWh})$ of the dispatchable generator, it still stays online and operates at a low level due to effects of startup and shut-down costs. The flexible load unit shifts its load from high price period to low price period, i.e. the load increases in hours 3-5 and decreases in hours 8, 9 and 20.

\section{Effects of Market Environments and Limited ID Trading}

Table I compares the expected revenue and the deviation considering different market environments. It can be seen that the deviation amount decreases and the expected revenue increases, as more trading opportunities are considered. In general, more trading floors provide market participants more trading opportunities. Furthermore, the fact that the ID market clears closer to the delivery time provides an opportunity for market participants to optimize their positions to reduce risks associated with uncertain variable generation outputs. In addition, cases with different ID market trading limits are compared. To be specific, for each scenario the energy traded in the intraday market for the scheduling horizon (i.e. 24 hours) is limited to $10 \%$ and $20 \%$ of the energy traded in the DA market under the corresponding scenario. As expected, relaxing the limit results in an increase on the aggregator revenue and a reduction on the imbalance deviation.

TABLE I: Effects of market environments

\begin{tabular}{|c|c|c|c|}
\hline Market environment & $\begin{array}{c}\text { ID trading } \\
\text { limit }\end{array}$ & $\begin{array}{c}\text { Expected aggregator } \\
\text { revenue [€] }\end{array}$ & $\begin{array}{c}\text { Imbalance } \\
\text { deviation } \\
\text { [MWh] }\end{array}$ \\
\hline $\begin{array}{c}\text { Day-ahead and } \\
\text { balancing market }\end{array}$ & $\mathrm{n} / \mathrm{a}$ & 21,715 & 40.1 \\
\hline $\begin{array}{c}\text { Day-ahead, intraday } \\
\text { and balancing market }\end{array}$ & unlimited & $\begin{array}{c}28,817 \\
(+33 \%)\end{array}$ & $\begin{array}{c}25.8 \\
(-36 \%)\end{array}$ \\
\hline $\begin{array}{c}\text { Day-ahead, intraday } \\
\text { and balancing market }\end{array}$ & $20 \%$ & $\begin{array}{c}24,019 \\
(+11 \%)\end{array}$ & $\begin{array}{c}28.0 \\
(-30 \%)\end{array}$ \\
\hline $\begin{array}{c}\text { Day-ahead, intraday } \\
\text { and balancing market }\end{array}$ & $10 \%$ & $\begin{array}{c}23,171 \\
(+7 \%)\end{array}$ & $\begin{array}{c}29.1 \\
(-28 \%)\end{array}$ \\
\hline
\end{tabular}

\section{Effects of Risk Control}

In Fig. 6, the effect of different values of the weighting factor $\beta$ on the expected aggregator revenue and the revenue for each scenario is investigated. It shows that the total aggregator revenue decreases and the CVaR increases as the risk exposure decreases. The trade-off between the risk exposure and revenue helps to hedge against uncertainty when participating in sequential electricity markets.

\section{CONCLUSION}

A stochastic programming methodology to derive optimal trading strategies for an aggregator participating in sequential electricity markets is presented in this paper. The coordination of different DERs realizes a higher flexibility provision, and the integration of sequential markets results in an increase in the total expected revenue, guaranteeing at the same time lower

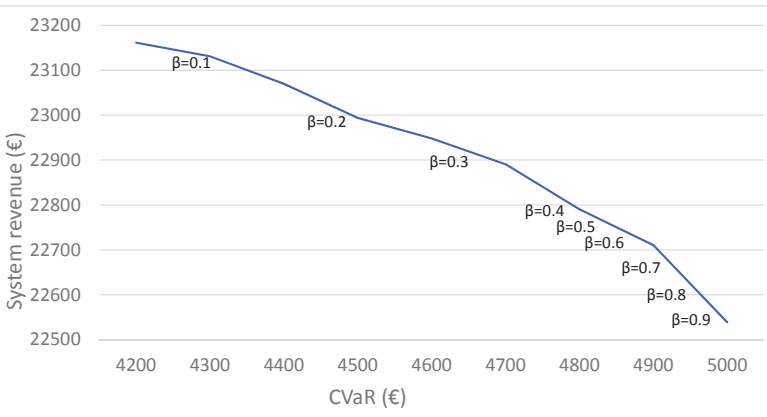

Fig. 6: Effects of risk control.

imbalances. In addition, the expected revenue is decreased and imbalance deviations are increased as a consequence of introducing limitations on the ID market capacity. Finally, risk control is conducted to assure a certain risk level when designing optimal trading strategies. Further analysis remains to be done on the frequency of modifying generation plans, i.e. the frequency at which ID auctions are executed.

\section{REFERENCES}

[1] E. G. Kardakos, C. K. Simoglou, and A. G. Bakirtzis, "Optimal bidding strategies of a mixed RES portfolio by stochastic programming," in Proc. IEEE PES Innovative Smart Grid Technologies, Europe, 2014.

[2] A. J. Conejo, J. M. Morales, and J. A. Martinez, "Tools for the analysis and design of distributed resources part III: Market studies," IEEE Transactions on Power Delivery, vol. 26, no. 3, pp. 1663-1670, 2011.

[3] J. M. Morales, A. J. Conejo, and J. Pérez-Ruiz, "Short-term trading for a wind power producer," IEEE Transactions on Power Systems, vol. 25, no. 1 , pp. 554-564, 2010.

[4] J. L. Angarita, J. Usaola, and J. Martínez-Crespo, "Combined hydrowind generation bids in a pool-based electricity market," Electric Power Systems Research, vol. 79, no. 7, pp. 1038-1046, 2009.

[5] S. Delikaraoglou, A. Papakonstantinou, C. Ordoudis, and P. Pinson, "Price-maker wind power producer participating in a joint day-ahead and real-time market," in Proc. 12th Conf. Eur. Energy Market (EEM), 2015.

[6] E. Faria and S.-E. Fleten, "Day-ahead market bidding for a Nordic hydropower producer: taking the Elbas market into account," Computational Management Science, vol. 8, no. 1-2, pp. 75-101, 2011.

[7] J. Garcia-Gonzalez, R. M. R. de la Muela, L. M. Santos, and A. M. Gonzalez, "Stochastic joint optimization of wind generation and pumpedstorage units in an electricity market," IEEE Transactions on Power Systems, vol. 23, no. 2, pp. 460-468, 2008.

[8] M. Peik-Herfeh, H. Seifi, and M. Sheikh-El-Eslami, "Decision making of a virtual power plant under uncertainties for bidding in a day-ahead market using point estimate method," International Journal of Electrical Power \& Energy Systems, vol. 44, no. 1, pp. 88-98, 2013.

[9] I. Kuzle, M. Zdrilić, and H. Pandžić, "Virtual power plant dispatch optimization using linear programming," in Proc. 10th Int. Conf. Environ. Elect. Eng. (EEEIC), 2011.

[10] H. Pandžić, J. M. Morales, A. J. Conejo, and I. Kuzle, "Offering model for a virtual power plant based on stochastic programming," Applied Energy, vol. 105, pp. 282-292, 2013.

[11] J. Abrell, "The Swiss wholesale electricity market," 2016.

[12] A. J. Conejo, M. Carrión, and J. M. Morales, Decision making under uncertainty in electricity markets. New York: Springer, 2010.

[13] E. G. Kardakos, C. K. Simoglou, and A. G. Bakirtzis, "Optimal offering strategy of a virtual power plant: A stochastic bi-level approach," IEEE Transactions on Smart Grid, vol. 7, no. 2, pp. 794-806, 2016.

[14] R. T. Rockafellar and S. Uryasev, "Optimization of conditional valueat-risk," Journal of risk, vol. 2, pp. 21-42, 2000.

[15] European Power Exchange, "EPEX market data [online]." Available: https://www.epexspot.com/en/market-data, Jan. 2017.

[16] I. Staffell and S. Pfenninger, "Using bias-corrected reanalysis to simulate current and future wind power output," Energy, vol. 114, pp. 1224-1239, 2016.

[17] S. Aventa AG, Leichtwindanlange AV-7. http://www.aventa.ch. 